\title{
The Role of Contextual Info-Marks in Navigating a Virtual Rural Environment
}

\author{
Adam Rousell, ${ }^{\dagger}$ Claire Jarvis ${ }^{\dagger}$ and Chris Brunsdon ${ }^{\ddagger}$ \\ ${ }^{+}$Department of Geography, University of Leicester \\ ${ }^{\ddagger}$ National Centre for Geocomputation, National University of Ireland, Maynooth
}

\begin{abstract}
Navigation is a task performed in both large and small scale environments. Landmarks within an environment are of great benefit to these navigational tasks, but in large rural environments such landmarks may be sparse. It has been shown that landmarks need not be purely visual and that a change in context for a feature can make it become a landmark against its surroundings (such as being provided with significant meaning). Such meaning could be added through personal experience or by informing the observer via some form of communication. To investigate the effects of providing such contextual information on navigational performance, experiments were conducted in a large rural virtual environment where the delivery method of the information was varied between onscreen and PDA display. Users were instructed to perform a route tracing navigation task. In some instances users were presented with textual information about specific locations within the environment which appeared when they were in the vicinity of the location. Both quantitative and qualitative data were collected and analyzed, with results indicating that although the actual performance in the task was not significantly improved, users felt that their performance was better and the task easier when they were presented with the contextual information.
\end{abstract}

\section{Introduction}

Navigation is essential in any environment that requires movement over a large distance (Darken and Peterson 2001) and in smaller and more confined spaces alike (e.g. Byagowi and Moussavi 2012; Ruddle et al. 2013; Thibault et al. 2013). In a number of instances, distinctive features can be used as landmarks to aid in these navigation processes. However, in environments where such features are widely scattered and intervisibility between them is low (it is often the case that from the location of one landmark, another is difficult to see), they become of little navigational use, as the user would not be able to determine a correct direction for travel using them. Montello (2005) uses the term "visual access" for this observational relationship between features, although in this study the term intervisibility is used as it implies a more interconnected relationship. With the wide use of technologies that provide information based on location and the integration of this information into georeferenced data, there is an opportunity for the digital domain to provide an extra level of navigational information that either increases the intervisibility of such landmarks or simply introduces new ones to the environment. Such pieces of information, attached to a geographic location and presented to a user, are referred to in this study as 'info-marks'.

\footnotetext{
Address for correspondence: Adam Rousell, GIScience Research Group, University of Heidelberg, Berliner Straße 48, 69120 Heidelberg. Germany. E-mail:adam.rousell@geog.uni-heidelberg.de

Acknowledgments: This work has been conducted as part of the first author's PhD studies which were funded by the University of Leicester. The authors would like to thank the anonymous reviewers for their helpful comments.
} 
Montello (2005) notes that the process of navigation is comprised of two distinct parts: locomotion (the actual movement in an environment), and wayfinding (the planning of a route around an environment). The aspect of navigation that is focused on in this article is wayfinding, in particular the concept of orientation which can be seen as maintaining a sense of where we are relative to the end goal (Montello 2005). This study involves the assessment of whether such info-marks actually have an impact on navigational performance. The research takes place in the context of a Virtual Environment (VE); skills gained in VEs can transfer to real world instances (Giudice et al. 2010). Implementation in a VE provides for a more controlled experimental environment and reduces the time needed for users to travel larger distances as speed can be easily increased within the VE. The basic VE implementation was enhanced through programmatical procedures to present the info-marks to the user either onscreen or via a PDA device. Additionally, the system kept track logs of user movements for later analysis. The presentation of information via both PDA and onscreen methods was implemented as a means of determining whether the actual implementation of the aids could have an effect on their usefulness. Such a difference (if found) would have an impact on any future development of tools as some are less practical in real world instances (the onscreen delivery method would not be possible in a real world instance). An additional note at this stage is the use of a rural setting for the experiment. A number of previous studies relating to navigation have been conducted in either urban or indoor settings whereby the well-structured layout of the environment restricts the movement of users. In a rural setting such structure is lacking and so people are given much more freedom in direction and paths that they can take. Such freedom could pose problems to navigational tasks as it results in a much wider field of movement. This in turn means that the probability of a person coming across a distinct landmark which could aid in their wayfinding tasks is drastically reduced. The lack of extensive research into navigation within such unstructured environments is a key gap in the wayfinding literature.

This article presents the rationale, methodology and findings of the experiment implemented to identify the effects of info-marks on navigational performance. The article first presents the findings of a literature review into the topics of navigation, VEs and Location- and Context-Based Services (LCBS). This provides the rationale behind the study. Next the methodology is presented consisting of the implementation of the VE and the info-marks as well as procedures used for information gathering. Finally, both qualitative and quantitative results and analysis are presented, before a summary of findings together with the implications for future research.

\section{Literature Review and Rationale}

VEs have been used in a number of instances to train people how to perform tasks that can be dangerous or difficult to perform in real world instances (e.g. Fillatreau et al. 2013; Lyu et al. 2013). They have also been used in a number of instances to investigate the effects of user navigation (Grant and Magee 1998; Lathrop and Kaiser 2005; Byagowi and Moussavi 2012). One reason behind this is that it has been shown that methods used for navigation in such VEs are similar to those methods used in real world environments (Aoki et al. 2008; Biggs et al. 2008; Sturz et al. 2009) and that skills gained in a VE are directly transferable to real world navigation (Giudice et al. 2010). A study by Koenig et al. (2011) showed that no significant difference was found between navigation performance in virtual and real world environments, although they do note that aspects such as simulation sickness (see also Vinson et al. 2012), display distortion and model detail cause problems for users of VEs and as such should be addressed in future research. 
The research domain of spatial knowledge is a well investigated topic with research dating back several decades (i.e. Siegel and White 1975; Lynch 1960). Such research tends to focus heavily on how information about an environment is stored in mental projections and how this information can be used for tasks such as navigation, orientation and wayfinding. Navigation can be seen as the moving between two locations, while orientation is the ability to determine one's location within an environment relative to other objects (Parush and Berman 2004). As such, orientation is a key aspect to being able to navigate successfully.

One predominant theory regarding the understanding of the spatial layout of an environment is that cognitive maps are generated by the perceiver. These maps are constructed using three main forms of knowledge: landmark, route and survey (Abu-Obeid 1998; Ruddle and Péruch 2004; Sjölinder et al. 2005). Landmark knowledge is information gained about distinctive environmental features (Abu-Obeid 1998). It is understood that such landmarks do not necessarily have to be singular distinct objects but can be items such as road junctions (Ruddle and Péruch 2004) or even meaningful events in time (Siegel and White 1975). Route knowledge refers to the linkages between environmental features (Abu-Obeid 1998) and is closely linked with the ability to navigate between locations (Ruddle and Péruch 2004). Survey knowledge is a more debated form of knowledge with regards to what it represents, but can generally be seen as a general global overview of the environment and allows for more flexible navigation between locations (Hurlebaus et al. 2008). Obviously, understanding the layout of an environment is essential for navigation and orientation and so the development of an effective cognitive map is key (Burigat and Chittaro 2007; Bosco et al. 2008).

Landmarks are an essential aspect for navigating in both virtual and real world environments (Lazem and Sheta 2005). Vinson (1999) suggests that within VEs, landmarks can be used as reference points for both navigation and orientation tasks. In their study, a number of guidelines are set out regarding the use of landmarks within VEs and how they should be implemented to improve performance in such tasks. These guidelines include making landmarks distinct, placing them at major decision-making points and making them easy to distinguish from other features in the environment. Several 'facts' relating to the nature of landmarks are also described in Klippel and Winter (2005) who include aspects such as the location of landmarks in relation to turning points and the importance of landmarks in finding one's way in an environment. Whereas a number of recent advances in human navigation focus on urban contexts, less research has been conducted within natural environments (Brosset et al. 2008). Montello (2005) discusses several aspects that make urban and natural environments different including the presence of more regular patterns in urban spaces (right angles and straight lines) and a greater level of visual homogeneity in rural landscapes. Kettunen et al. (2013) investigate the effects of seasons on the salience of different types of landmarks in natural environments. Results indicated that features such as passages, rock formations and trees were all used but with varying levels between winter and summer months. Again in that study however, only visible features were used as landmarks. Snowdon and Kray (2009) also highlight the importance of season and time of day when using visible landmarks in natural environments and state that any service that uses photography as a means of aiding navigation would need to take such aspects into account. This again suggests that a method which does not rely purely on visual information may be preferable with regards to providing navigation assistance within such natural landscapes. Away from navigation in geographic environments, Sorrows and Hirtle (1999) discuss the concept of landmarks in cyber-space where items such as navigation bars, FAQ pages and index pages can be seen as landmarks. This, along with the concept of temporal events creating landmarks as described by Siegel and 
White (1975), highlights that landmarks may not necessarily have to be physical features to be useful. Such abstract landmarks can also be used by electronic devices (i.e. infrared beacons) to provide navigational cues (Raubal and Winter 2002) to their user. Overall, it is clear that landmarks can be formed from a number of features including distinct objects, intersections of roads and distinct anchor points in cyber navigation.

A key characteristic of landmarks is that in large environments not all landmarks will be visible at any one time. Pierce and Pausch (2004) assessed benefits of providing landmarks that are visible at all scales when moving within a VE. Results from that study indicated that providing landmarks across all scales made traversing large virtual worlds more efficient. In addition, Rousell et al. (2008) found that the provision of global landmarks indicating the location of urbanization in a large VE aided in determining a user's current location.

As a means of addressing the common problem of identifying what might best be used as a landmark, Raubal and Winter (2002) propose a method for deriving objects to represent as landmarks in a navigation service. This method used a number of feature attributes (relating to appearance and semantic relationships) to identify which objects would be most useful as a landmark. One issue with their implementation was that an assumption was made that, when moving between nodes in a navigational grid, users do not require any landmarks other than to inform them of when they arrive at the nodes (i.e. possible turning points). Although this is likely to be true in well structured environments where the transition between nodes is well defined (i.e. to travel between road junctions in a city you cannot deviate from the roads), in open areas such as rural environments such deviations are likely. This means that only having landmarks at key turning points along a path would not be of much benefit; the user would deviate from the straight line and so miss the turning points (and so the landmarks).

As mentioned, electronic landmarks can be used by devices for navigational tasks. A number of electronic resources are now being geo-tagged with a location which is related to the contents of the resource (Yaegashi and Yanai 2009). Such geo-tagged resources can be photographs, textual descriptive or audio recordings. Another aspect of electronic devices linking with real world locations is the field of Location- and Context-Based Services (LCBS). These are computerized services which have an inherent link between an application and some form of location or activity context (Kühn 2004). Examples of LCBS include transport logistics for monitoring the location of goods, automatic collision avoidance systems, and the coordination of actions in a rescue or emergency support task. A similar concept to LCBS is that of Interaction Loci which refers to the presentation of information to a user based on location. Fogli et al. (2003) used Interaction Loci to present visitors to a museum with information about exhibits via a mobile device based on the visitor's location. Ardito et al. (2007) used a similar concept but the user was immersed in a VE. Both studies highlight that beneficial methods can be implemented to present useful information to users based on their location; information that may not be readily apparent normally.

From the literature it is clear that navigation is an important task to be performed within both real and virtual environments. It has also been shown that navigational tasks within a VE maps to similar tasks in the real world in terms of methods used and so VEs are an ideal testbed for implementing research into ways of improving navigational performance. One key aspect that influences performance in navigational tasks is that of landmark availability. If an environment has few landmarks, then navigation and orientation proves difficult, especially in rural areas where landscapes can appear relatively monotonous and movement is not restricted by corridors created by physical features. Research has shown that landmarks do not necessarily need to be physical to be useful, and that LCBS and geo-tagging of electronic resources is becoming more widely used. As such, it is possible that items that are not directly visible 
could become useful to navigators if they are made aware of their presence by such LCBS technologies. In addition, non-distinct features or areas could possibly be made distinctive by giving context to them, which again could be delivered by such LCBS technologies. By linking information and data to a location in physical or virtual space and then presenting this to a user when they are within a certain distance from it, navigation could be improved. This is due to the information and its display to the user creating a contextual link to a physical location within the environment. This linkage of information and feature can be seen as an info-mark a landmark that is contextually information based. This study investigates areas that have been predominantly neglected in the research field as a whole. Firstly, most research into navigation has taken place in structured (i.e. urban) environments or indoor environments. In such structured environments, users can only travel along pre-defined corridors (i.e. roads) and it is often the case that landmarks fall within the visibility of such corridors thus facilitating the use of such landmarks for navigation tasks. While these are areas that many people navigate within, so the research is by no means mis-focused, regions that are larger and less structured are likely to present different navigational issues such as the intervisibility (the ability to view other landmarks from one that the observer is located at) of landmarks and the freedom of movement. Indeed, path-like features which can be used to direct movements are still present in the environment, but the user does not have to follow them as vigorously as they would in an urban environment as there are far fewer physical barriers that force them to these corridors. In the instance of this investigation, a method of locomotion similar to walking (albeit at a greater velocity) is implemented meaning that there is no particular movement restriction imposed by a motor vehicle (for example). Secondly, a number of studies focus on visually emphasizing existing landmarks or creating new ones that could aid in navigation. In a real world setting, the creation of landmarks may be impractical, and due to the large distances involved in rural environments, visually enhancing the existing ones may prove of little use. Therefore, this study focuses on a separate yet linked direction - using additional information to allow existing features to become more like global landmarks by presenting information relating to them when a person is within their vicinity, even though the feature may not be directly visible.

\section{Methodology}

The primary objective of this study was to assess whether the presentation of info-marks could aid in navigational performance in areas with few physical landmarks. The test-bed for the implementation and investigation of the effects of presenting users with info-marks was a VE. This environment was a realistic representation of the Sorbas region in south-eastern Spain. The reason that this area was selected was because it was used as a field trip site for geography undergraduate students from the University of Leicester, UK. By implementing the study in a VE, a number of methods could be used to collect data and experiments could be performed from afar.

\subsection{Virtual Environment and Info-Mark Delivery}

The VE was constructed from GIS datasets including road and river networks, building footprints, land cover classifications and a DEM (Digital Elevation Model, obtained from the Junta de Andalucía). The environment was rural in nature, comprising a number of different land cover classifications ranging from small urban settlements to dense natural vegetation. 
Features such as rivers, roads and fences were also present in the environment, although the dispersal of these was variable. Topography of the environment ranged from plateaus and valleys to fairly steep elevated regions. This information was fed into the Bionatics Blueberry procedural terrain generator software and representations for vegetation and man-made structures created. The environment was run in a specialist VR theatre using stereo imagery projected onto a large projector screen. Movement within the environment was controlled using a computer mouse and keyboard setup with a maximum travelling velocity of $30 \mathrm{~m} / \mathrm{s}$. The actual travelling speed could be varied by the user via system input (the wheel button on the mouse) but could not rise above this value. A compass was also integrated onto the display as a simple aid to enable determination of direction. The primary rationale for site selection was that the location is used as a field study site for second-year undergraduate geography students at the University of Leicester. Not only did this mean that data were already available, it was also hoped that the developed environment could be used as a pre-visit training tool for the students to familiarize themselves with the location. In theory, any environment could have been used for this investigation, but the primary focus of this study is to investigate the effectiveness of the navigation tool provided with the possible outcome that any benefits seen by using the tool in the VE could transfer to a real-life implementation in the same physical environment.

The info-marks themselves were based on textual information obtained from a number of sources. These texts related to 'interesting' characteristics or features of the area such as a description of a local church or some information relating to geological features present in particular areas. The purpose of this descriptive piece of information is to add context to the area or feature of interest which may otherwise be overlooked (i.e. the history of a building). Each info-mark was comprised of a title (the name of the feature) and a short textual description. This information was presented to the user either on-screen in the VE or via a PDA device when they were within one kilometer of the feature being described (in the case of non-point features, a centroid was used). This distance was selected to ensure a wide coverage of the environment with the info-mark regions. The info-mark areas were derived using a buffer of $1 \mathrm{~km}$ around each point and a Voronoi partition of the whole region based on the info-mark points. The buffers were then clipped using the Voronoi polygons to ensure that in the case of more than one point being within $1 \mathrm{~km}$, the info-mark information was presented for the point the user was closest to.

As mentioned, the info-marks were delivered in two methods: via a PDA, or displayed on-screen with the environment itself. The reason for using these two different delivery methods was to identify if there was the possibility that the delivery method of the information influenced how useful the info-marks actually were. In addition, if the same info-mark aid for navigation was to be used in the real world, the delivery method would likely be via the PDA whereas on a simple desktop implementation the onscreen method would be more practical. On-screen, an information box appeared in the top right corner of the interface. The PDA deployment was implemented using the HP MScape software (http://www.hpl.hp.com/ downloads/mediascape/) running on the PDA device which uses scripts and location-based sensors to present users with various media when the device is in a specific location. The VE system transmitted a Bluetooth signal imitating an external GPS receiver which was detected by the PDA and interpreted as indicating a location of the real-world counterpart in Spain. This meant that although the user and the device remained stationary, movements within the VE resulted in a different location being transmitted throughout the navigational task. As the position obtained from the computer system was exact (explicit coordinates of the user could be obtained in easting and northing values which were then transformed to latitude/longitude 


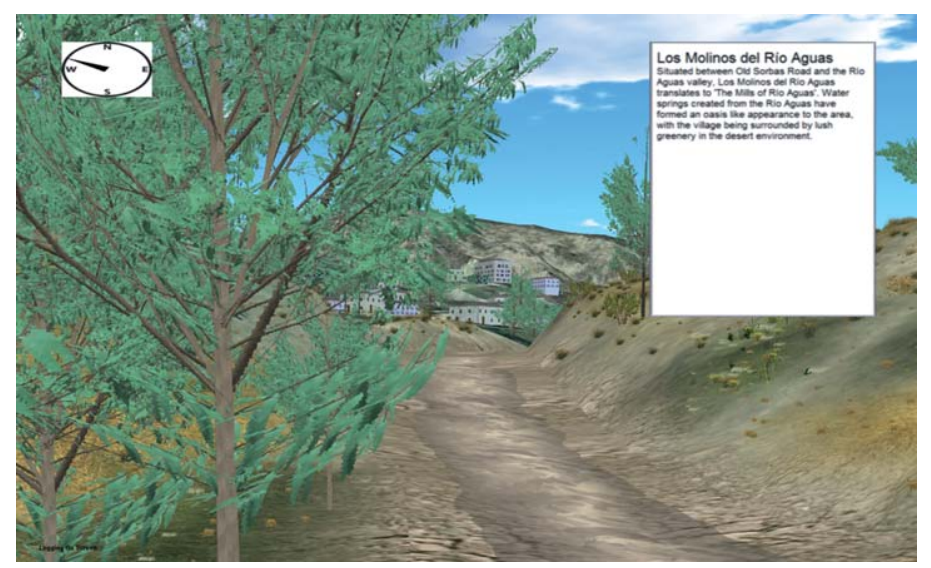

Figure 1 Screen shot of VE showing the on-screen info-mark display

values), the GPS positional accuracy of the PDA could be seen as being perfect, with the only spatial errors occurring due to transmission artifacts or the conversion process to latitude and longitude. Checksum validation was performed by the device on 'GPS' sentences received to ensure that the sentences corrupted during transmission were not incorporated into the position determination. Figure 1 shows the environment and an on-screen info-mark display.

A primary benefit of using the PDA delivery method is that all information about the infomarks is contained on the device and is free from the VE implementation. This means that not only can they be changed quickly without knowledge of the VE, they can also be used in the real world location that the VE simulates without modification. This is of particular advantage in the case of this study, as it would be possible for the info-marks to be used during the undergraduate field trip, as mentioned earlier.

\subsection{Experimental Procedure}

To assess the effects of the info-marks on navigational performance, a series of user trials were conducted. Each task consisted of a route-tracing exercise with data collected from track logs, from trial verbal reporting and from post-trial semi-structured interviews. Ethical clearance was obtained from the University of Leicester Ethics Board and all participants signed an informed consent form. Before beginning the experiment all users entered a training VE where they were instructed to move around so that they became familiar with the controls. When they reported they were comfortable with the interface the test environment was loaded and the experiment began. The time required to feel comfortable varied between participants, but was generally between $5-10$ minutes.

Thirty participants took part in the experiment with 10 in each info-mark delivery method group. The subjects were recruited from the University of Leicester and consisted predominantly of staff and students from the Department of Geography as well as other members of staff from other university areas. The participants consisted of 12 males and 18 females, with an average age of 29 based on age groups identified in post-trial interviews. All users were asked to describe how they perceived their skills with maps and navigation via a scaled metric (1-10 with 1 being a low self-perceived skill and 10 being high). Although tests such as the 
Santa Barbara Sense of Direction (Hegarty et al. 2002) exist and are well documented, for this investigation it was felt that more lightweight and direct questioning would be preferable. By directly asking the participants their perceived skill, the time required for completing the posttrial interview was reduced and a more personal and general self-perception metric obtained. The purpose of asking for self-perceived skill in this investigation was simply to identify a general level of ability between users. The more pertinent self-perceived questions related directly to the task itself (perceived ease and accuracy) and were used to identify a correlation between how the user felt and how they actually performed. Such questions linked directly to the task itself are not the target of the Santa Barbara Sense of Direction questionnaire. From the feedback for the general personal skill questioning, the average self-reported skill with maps was 6.97 out of 10 with a standard deviation of 1.45 . Self-reported navigation skill had an average of 6.37 out of 10 with a standard deviation of 1.85 . All subjects had normal or corrected to normal vision. An incentive of entry into a prize draw to win a book voucher was given to all users.

Three groups of testing were conducted in the experiment as a whole which represent different portrayal of the info-marks. For one group no info-marks were displayed at all. In the other two groups the portrayal method of the info-marks was altered with one group having the information displayed on screen in the VE interface and the other having it delivered via a PDA device (DELL AXIM X51V).

The navigation task itself was a route tracing exercise. The user was presented with a paper map (see Figure 2) of the environment showing features such as roads, rivers, contours and buildings. A route was depicted on the map and the user was instructed to trace that route as accurately as they could in the environment. The route was designed to cover a number of different feature types and land cover classifications, as well as ensuring that it entered a number of regions where the textual info-mark information would be displayed. Decision points on the route were not placed at distinctive environmental features as the purpose of the study is the use of the info-marks in an unstructured (i.e. few distinctive features) environment. If the decision points were placed at such distinctive features, then the navigation and orientation methods used would be likely to focus on those features as opposed to the areal info-mark data. In that instance, the results obtained would not necessarily be a suitable representation of the use of info-marks when other landmark features are not present. Obviously it is likely that in real world instances users would never have to turn in a location in the middle of nowhere, but it may be the case that turning points occur in areas where there are few landmarks to identify turning points along a feature (i.e. a long road). In that case, additional information in the form of info-marks could be highly beneficial to reduce the amount of uncertainty. When they perceived they were at the finishing location they were told to inform the researcher and the navigational task would end. Also displayed on the map were the locations that the info-marks were relating to. The same map was provided in all trials whether info-marks were to be made available or not, and the starting location in the environment was the starting location of the route.

Users were instructed to verbalize their thoughts and decisions during the task so that these could be recorded and analyzed. After the navigation task was completed a semistructured interview was conducted in an attempt to understand aspects relating to the user's self-perceived performance and to investigate any interesting observations made by the researcher during the experiment. Questions included the identification of areas that were particularly difficult or easy to navigate and what information in the map and environment were used. Questions were also asked relating to self-rating of perceived skill with map use and navigation, ease of the task, and how accurate the users perceived themselves to be in 


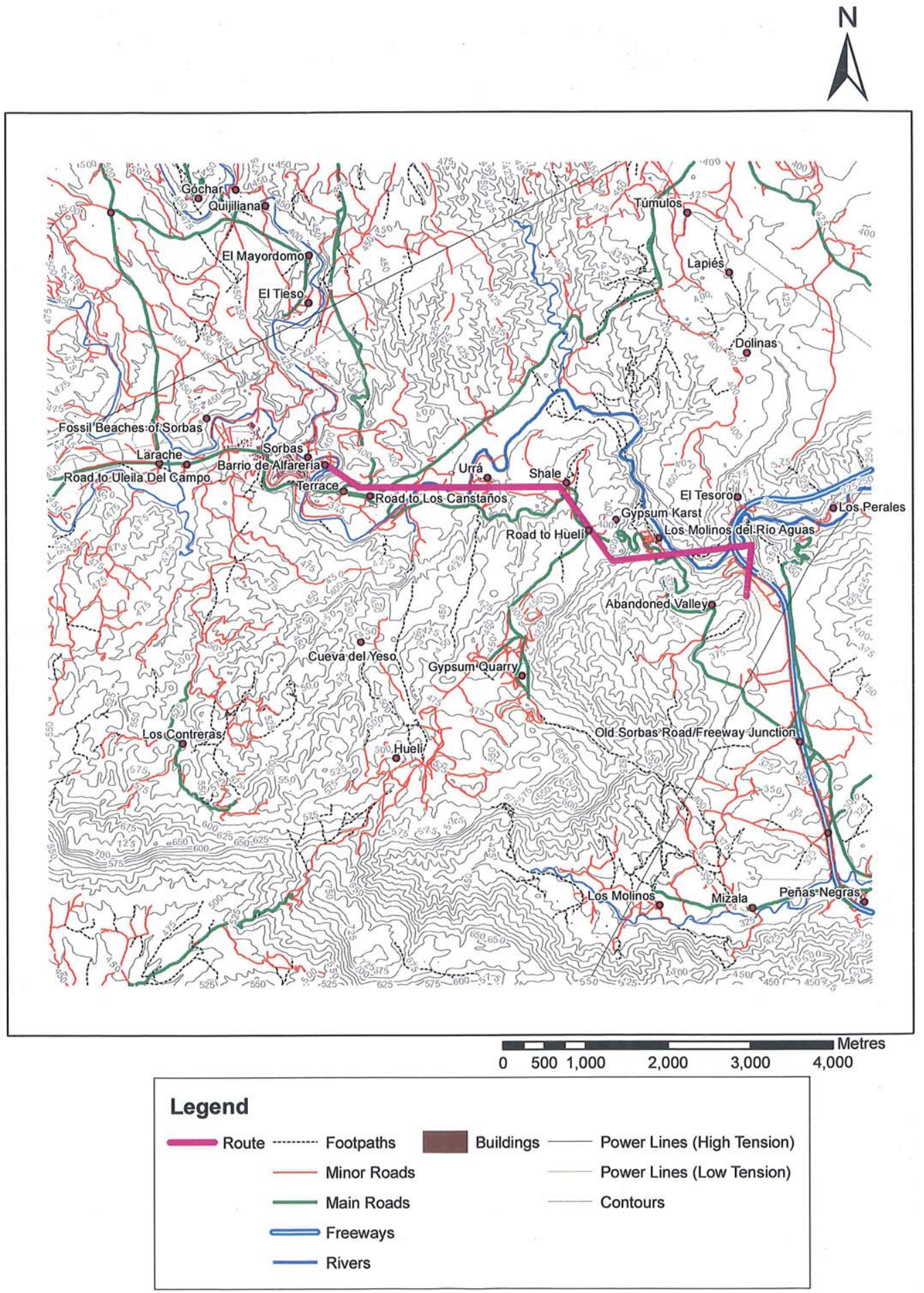

Figure 2 Map presented to users showing the intended route 


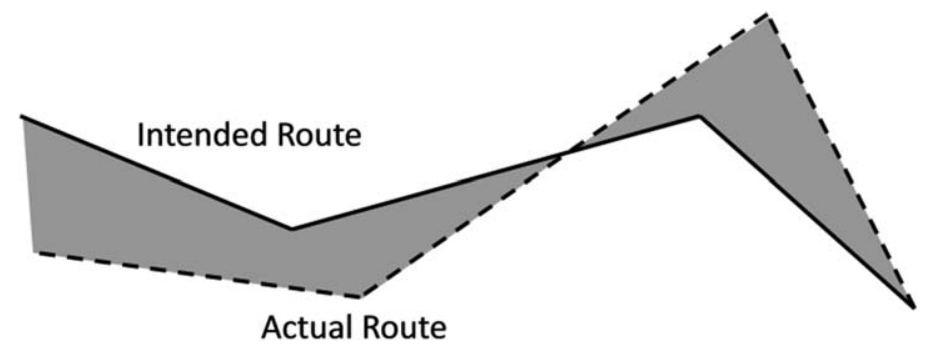

Figure 3 Determination of the deviation metric

following the defined route. Qualitative analysis was performed on transcripts for the in-trial verbal reporting and post-trial interviews. Trials were recorded using a dictaphone (if agreed at the informed consent stage) and these audio files were used for manual transcription.

Quantitative data were collected by maintaining a track log of user position and rotation throughout the experiment. These track logs were imported into GIS software and information was extracted relating to deviation from intended route, duration of experiment, distance travelled, average speed and distance from end point. The deviation metric was obtained by determining the area of polygons that formed between the intended and actual route (Figure 3). The other metrics mentioned were obtained through simple arithmetic calculations and standard GIS techniques.

To gain a general overview of user performance, a combined metric was also calculated. This was generated by determining a relational value for each metric against the global values for that metric and then summing across all metrics:

$$
m_{g}=\sum\left(\frac{m_{n}-\bar{m}_{n}}{\sigma_{m n}}\right)
$$

where $m_{g}$ is the global metric for the individual track log, $m_{n}$ is the track log value for metric $n, \bar{m}_{\mathrm{n}}$ is the mean of the metric $n$ across all track logs, and $\sigma_{m n}$ is the standard deviation of metric $n$ across all track logs.

\section{Results}

Results were obtained from the experiment in both qualitative and quantitative formats. Qualitative data were extracted from transcripts of in-trial feedback and post-trial interviews, and the quantitative data obtained from the track log data recorded by the system.

\subsection{Quantitative Analyses}

Qualitative analysis was performed using the metrics described in the experimental procedure section. Preliminary testing using Kolmorogov-Smirnov testing identified that all groups can be seen as representative of $\mathrm{t}$-distributions (see Table 1). All tests had a df value of nine.

To test for significance in the metrics between groups, Welch two sample t-tests were used. The primary reason for using t-tests was that they allow for comparison between groups within the metrics (none vs. on-screen, on-screen vs. PDA, and none vs. PDA) as opposed to seeing if one group was different from the overall population. In addition, the Welch test does 
Table 1 Results of KS-test

\begin{tabular}{|c|c|c|c|c|c|c|}
\hline & Area $\left(\mathrm{m}^{2}\right)$ & Length (m) & Distance (m) & Combined & Duration (s) & Speed $(\mathrm{m} / \mathrm{s})$ \\
\hline \multicolumn{7}{|c|}{ None } \\
\hline Mean & $1,279,047$ & 7121.883 & 566.7073 & 0.240417 & $1,283.1$ & 5.844603 \\
\hline SD & $1,019,232$ & 1757.077 & 900.7383 & 3.433075 & 397.3925 & 1.175863 \\
\hline $\mathrm{D}$ & 0.5 & 0.3 & 0.4 & 0.3 & 0.4 & 0.5 \\
\hline $\mathrm{p}$ & 0.1678 & 0.7869 & 0.4175 & 0.7869 & 0.4175 & 0.1678 \\
\hline \multicolumn{7}{|c|}{ On Screen } \\
\hline Mean & $1,168,536$ & 8112.402 & 196.6794 & -0.1468 & $1,315.9$ & 6.676225 \\
\hline $\mathrm{SD}$ & $725,312.4$ & 1201.206 & 163.8182 & 1.648741 & 431.6275 & 2.202596 \\
\hline $\mathrm{D}$ & 0.3 & 0.6 & 0.3 & 0.2 & 0.3 & 0.2 \\
\hline $\mathrm{p}$ & 0.7869 & 0.0525 & 0.7869 & 0.9945 & 0.7869 & 0.9945 \\
\hline \multicolumn{7}{|c|}{ PDA } \\
\hline Mean & $1,236,381$ & 7634.694 & 355.8074 & -0.09361 & $1,115.9$ & 7.089421 \\
\hline $\mathrm{SD}$ & $744,026.1$ & 2692.204 & 353.3158 & 3.117879 & 477.353 & 1.332448 \\
\hline $\mathrm{D}$ & 0.3 & 0.3 & 0.3 & 0.6 & 0.3 & 0.2 \\
\hline $\mathrm{p}$ & 0.7869 & 0.7869 & 0.7869 & 0.0525 & 0.7869 & 0.9945 \\
\hline
\end{tabular}

not assume equal variance as an ANOVA does. Results from the t-tests (at the $95 \%$ confidence level) can be seen in Table 2. From the results obtained it was apparent that the only instance where a significant difference was detected was between the no info-mark and PDA display groups within the average travel speed metric $(t=-2.213, \mathrm{p}=0.04027)$.

\subsection{Qualitative Analysis}

Qualitative analysis was performed using data obtained from the in-trial reporting and post trial interviews.

\subsubsection{In-trial reporting}

Any mentions of particular features or feature types in the in-trial reporting were counted as a means of identifying which features were used more in the navigational task. No distinction was made between whether the features were mentioned in a positive or negative context as it was deemed that any mention implies that they were being used in some form. These counts were then converted into percentages of total feature mentions with results being seen in Table 3.

From these results it is clear that when no info-marks were presented to the user, the road and river features were heavily used in the navigation tasks. In two instances the info-marks were mentioned although they were not displayed as the user interpreted them from the map provided, and the users were informed at the beginning of the trial that those items on the map might not appear in the environment. When info-marks were made available they were, unsurprisingly, mentioned during the trials, indicating that in some way they were used. However, they were mentioned more when presented via the PDA than when they were 
Table 2 T-test $\mathrm{p}$ values for comparison of metrics between info-mark display methods

\begin{tabular}{llccc}
\hline Metric & & None vs. On-screen & None vs. PDA & On-screen vs. PDA \\
\hline \multirow{2}{*}{ Area } & $\mathrm{t}$ & 0.2794 & 0.1069 & -0.2065 \\
& $\mathrm{df}$ & 16.255 & 16.471 & 17.988 \\
\multirow{5}{*}{ Length } & $\mathrm{p}$ & 0.7835 & 0.9161 & 0.8387 \\
& $\mathrm{t}$ & -1.4716 & -0.5044 & 0.5124 \\
& $\mathrm{df}$ & 15.904 & 15.49 & 12.447 \\
Duration & $\mathrm{p}$ & 0.1606 & 0.6211 & 0.6173 \\
& $\mathrm{t}$ & -0.1768 & 0.8513 & 0.9827 \\
\multirow{3}{*}{ Speed } & $\mathrm{df}$ & 17.878 & 17.427 & 17.821 \\
& $\mathrm{p}$ & 0.8617 & 0.4062 & 0.3389 \\
& $\mathrm{t}$ & -1.0494 & -2.213 & -0.5095 \\
Distance & $\mathrm{df}$ & 13.74 & 17.724 & 14.807 \\
& $\mathrm{p}$ & 0.3121 & 0.04027 & 0.6179 \\
& $\mathrm{t}$ & 1.2781 & 0.6893 & -1.2921 \\
Combined & $\mathrm{df}$ & 9.595 & 11.705 & 12.699 \\
& $\mathrm{p}$ & 0.2313 & 0.5041 & 0.2193 \\
& $\mathrm{t}$ & 0.3214 & 0.2291 & -0.0457 \\
& $\mathrm{df}$ & 12.936 & 17.837 & 13.658 \\
& $\mathrm{p}$ & 0.753 & 0.8214 & 0.9642 \\
\hline
\end{tabular}

Table 3 Features identified for aiding in navigation

\begin{tabular}{llll}
\hline & None & On-screen & PDA \\
\hline 1st & Roads $(39 \%)$ & Roads $(49 \%)$ & Roads $(35 \%)$ \\
2nd & Rivers $(31 \%)$ & Compass bearings $(21 \%)$ & Info-marks $(34 \%)$ \\
3rd & Compass bearings $(13 \%)$ & Info-marks $(14 \%)$ & Compass bearings $(16 \%)$ \\
4th & Buildings $(10 \%)$ & Rivers $(12 \%)$ & Rivers $(9 \%)$ \\
5th & Terrain $(5 \%)$ & Terrain $(4 \%)$ & Terrain $(3 \%)$ \\
6th & Info-marks $(1 \%)$ & Buildings $(\sim 0 \%)$ & Buildings $(3 \%)$ \\
\hline
\end{tabular}

displayed on-screen. In both cases, roads were still the predominantly mentioned feature during the trials and so can be seen as the primary aid in navigation.

\subsubsection{Post-trial interviews}

The information gathered from post-trial interviews focused on how the user perceived the task and their performance, what context the info-marks were used in, and any improvements that could be made to make the task easier.

Users were asked to rate how easy they found the task and how accurate they perceived themselves to be on a scale of 1 to 10 , with 1 being very hard or highly inaccurate and 10 
Table 4 Ease of task and accuracy ratings

\begin{tabular}{|c|c|c|c|c|c|}
\hline \multicolumn{3}{|c|}{ Ease } & \multicolumn{3}{|c|}{ Accuracy } \\
\hline PDA & Screen & None & PDA & Screen & None \\
\hline 8 & 5 & 6 & 4 & 3 & 5 \\
\hline 7 & 8 & 7 & 7 & 6 & 5 \\
\hline 4 & 6 & 6 & 6 & 7 & 3 \\
\hline 8 & 4 & 5 & 7 & 6 & 7 \\
\hline 7 & 4 & 3 & 7 & 6 & 7 \\
\hline 5 & 6 & 5 & 8 & 4 & 3 \\
\hline 7 & 6 & 2 & 6 & 7 & 2 \\
\hline 5 & 5 & 2 & 8 & 6 & 6 \\
\hline \multirow[t]{2}{*}{4} & 4 & 3 & 7 & 5 & 3 \\
\hline & 8 & 3 & 6 & 6 & 4 \\
\hline
\end{tabular}

$1=$ very difficult/highly inaccurate and $10=$ very easy/highly accurate

Table 5 Ease of task and accuracy rating p-values

\begin{tabular}{lcc}
\hline & Ease & Accuracy \\
\hline None vs. onscreen & 0.1156 & 0.1638 \\
None vs. PDA & 0.0388 & 0.0122 \\
Onscreen vs. PDA & 0.5315 & 0.0624 \\
\hline
\end{tabular}

being very easy or highly accurate. Only nine values were obtained for the ease of task question for PDA delivery due to an omitted result during the interview process. Mann-Whitney tests were conducted for each measurement between the different info-mark groups. Results indicated that when users were presented with the info-marks they felt that the task was easier and that they were more accurate than when no info-marks were presented ( $\mathrm{p}=0.039$ and 0.012 , respectively). The values obtained from the ratings can be seen in Table 4 and the results of the Mann-Whitney tests in Table 5.

Another aspect addressed in the interviews was how the info-marks were used. This question addressed whether the info-marks were used more (in the context of landmarks) as an indication of following the correct route or for determining location. Only one user stated that the info-marks were not useful at all. Of the other responses, two did not identify how they were used, five users identified them as being mostly used for route confirmation, one as purely for determination of location, and seven users identified a mixture of the two.

\subsection{Comparison of Qualitative and Quantitative Results}

As a final piece of analysis, the results from the ease of task and accuracy rating questions were compared with the performance metrics gained from the track logs. The purpose of this was to identify whether there was any correlation between the metrics obtained in the trials 
Table 6 Spearman test results

\begin{tabular}{lrrrrr}
\hline & \multicolumn{2}{c}{ Ease } & & \multicolumn{2}{c}{ Accuracy } \\
\cline { 2 - 3 } \cline { 5 - 6 } & rho & $\mathrm{p}$ & & rho & $\mathrm{P}$ \\
\hline Area & -0.402 & 0.030 & -0.163 & 0.389 \\
Length & -0.320 & 0.090 & -0.444 & 0.014 \\
Duration & -0.414 & 0.026 & -0.099 & 0.604 \\
Speed & 0.299 & 0.116 & 0.025 & 0.895 \\
Distance & 0.194 & 0.314 & -0.110 & 0.563 \\
Combined & -0.463 & 0.011 & -0.443 & 0.014 \\
\hline
\end{tabular}

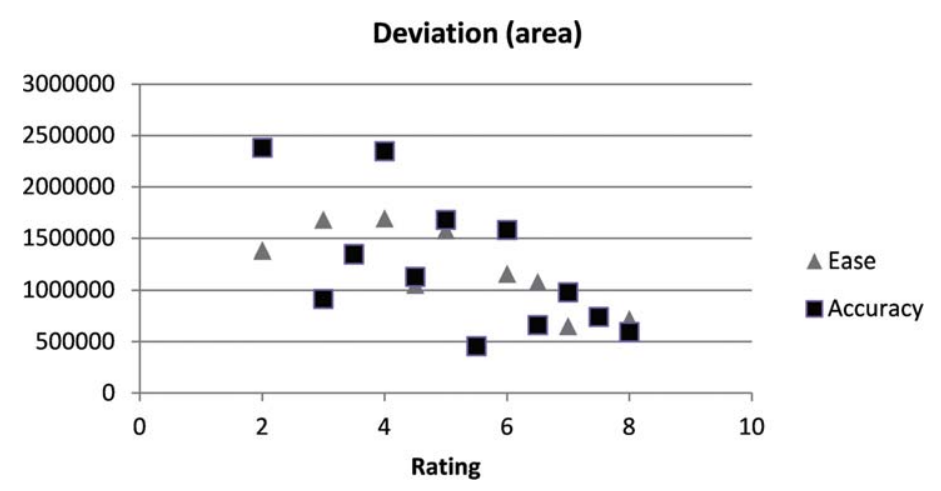

Figure 4 Plot of deviation metric against self perceived ease and accuracy ratings

and the users' perceived ease and accuracy. To investigate this, ranked Spearman tests were implemented between the qualitative assessments and metrics obtained from the track logs. A significance threshold was selected at the $95 \%$ confidence level using a two-tailed test with $\mathrm{df}=28$ for accuracy and $\mathrm{df}=27$ for ease of task (Table 6). Significant results were detected between the accuracy value and the deviation from route metric ( rho $=-0.444, p=0.014$ ), accuracy and combined metric (rho $=-0.443, \mathrm{p}=0.014)$ and the ease of task and combined metric (rho $=-0.463, \mathrm{p}=0.011$ ). These statistical significant results all showed a negative correlation between the qualitative rating and the metric meaning that as the rating increased, the metric decreased. This indicates that when the user perceived they were more accurate, their deviation from the intended route and the general combined metric was lower (Figures 4 and 5). Also, when they perceived the task was easier, the general performance metric decreased, indicating that they did actually perform better. No distinction was made in this analysis between the different delivery methods, as very little significance was found between the delivery methods during the earlier quantitative analysis.

\section{Discussion}

The purpose of this study was to ascertain whether the use of info-marks in a rural VE would assist in navigational performance. Although the quantitative analysis of track log data 


\section{Combined}

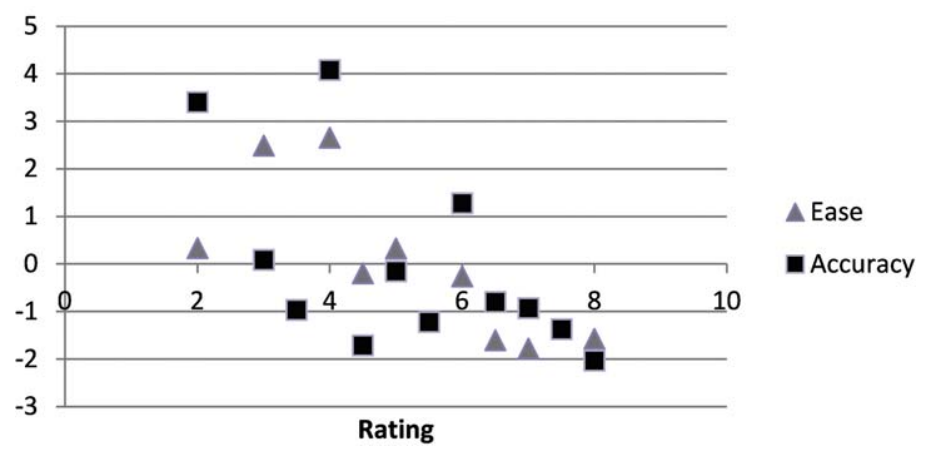

Figure 5 Plot of combined metric against self perceived ease and accuracy ratings

obtained from users undertaking a route tracing exercise showed little in the way of effect, qualitative feedback shows that perceived performance was altered. From a quantitative point of view, the only significant result was with respect to average travel speed where users were presented with info-marks via a PDA they completed the task at a faster speed. All other metrics were not affected by the presence of info-marks in either delivery method. There was a correlation found between a number of the metrics obtained from the track logs and the selfperceived ease of tasks and accuracy metrics, indicating that when users felt they were more accurate or the task was easier, they did indeed perform better in some of the metrics.

Information gathered from post-trial interviews highlighted that when info-marks were presented via a PDA device, users felt the task was easier and that they followed the intended route more accurately. According to the track log analysis, however, this was not the case. Therefore one of the primary outcomes of this study was not the effectiveness of the infomarks on navigational task performance but the importance of performing both quantitative and qualitative investigative methods. The reasoning behind this contradiction in results from the two different approaches could be that the presentation of the info-marks altered the methods used for navigation, but that these new approaches were no more accurate but were easier to perform. These differences in navigation methods could be the result of the user making use of the info-mark information to aid in navigation, but due to the information appearing up to a kilometer away from the feature itself, the amount of locational error introduced was greatly increased, as opposed to if it was presented at a smaller distance, such as $200 \mathrm{~m}$. Such changes in size were outside of the scope of this study but may warrant further investigation. When the information was not presented, or was presented via the onscreen delivery, the users relied solely on matching topographic features with the map, which could introduce a large amount of spatial uncertainty. In the in-trial feedback, users reported a high reliance on the info-marks for navigation, and in the post-trial interviews these were highlighted as being predominantly a cue as to whether the user was travelling in the correct direction. As the info-marks were areal in nature as opposed to specific points, the inaccuracies in performance could be accounted for by the inherent uncertainty as to where the feature was in relation to the user's position.

With regards to the different delivery methods, studies (i.e. Rashid et al. 2012a, b) have highlighted that switching between displays can have a cognitive 'cost' associated which in turn can cause a drop in performance. This cost is likely to be present in the results obtained from this study, as in both cases the user needed to shift their attention away from the VE. In 
the case of the PDA, the user often had to move their gaze completely away from the VE screen, but even with the on-screen display, where the information was shown within the same view region, the user still had to shift their attention to a sub window. However, as the on-screen display is in the same field of view, this cost could be less than when the user changed their view to look at the PDA, which often lay flat on a table by the keyboard and mouse (Cauchard et al. 2011). In this study however, little evidence has been seen that there is a significant difference between the PDA and onscreen delivery, with no significant results being found between PDA and onscreen groups.

Another aspect highlighted from the results is that when presented on screen the infomarks did not appear to have the same impact on perception of performance. It was also seen that the info-marks were not mentioned as much during the trial as they were when presented via the PDA. This poses the question as to what it is about the PDA delivery method that made it more 'useful'. Without further investigation it is not clear what the primary cause of this difference is. However, there are concepts that could be theorized. Firstly, when a new info-mark was displayed on the PDA a sound was played to draw the user's attention to this, as they would normally be looking at the screen and not the device. It may be the case that users either did not notice the change when presented onscreen, or that they simply paid less attention. Secondly, an effect may have been caused by the drawing of attention away from the environment whilst looking at the PDA device, such as a break in thought process that allows for the integration of the info-mark knowledge into the navigational processing.

In summary, although little quantifiable benefit was found to using info-marks in navigational tasks, this study has highlighted the importance of the use of qualitative and quantitative analysis when attempting to understand performance with relation to such tasks. To date, it is still often the case that when navigational performance is investigated, only the metrics obtained from track logs or techniques such as pointing are used for assessing performance changes. The self-perceived change in performance by the test subject is seldom included in analysis. Only by assessing both aspects can the concept that the info-marks altered navigation methods but with no actual performance benefits be achieved. As discussed, when comparing the two sets of results it could be hypothesized that by providing the areal info-marks users had an additional piece of information which could be used for determining route. However, due to the inherent uncertainty relating to the actual position in relation to the info-mark feature, such information could be misleading, so although making the task easier they do not make it more accurate. One method that could be implemented, which might improve this (without adding additional visuals to the environment itself), could be to give a sense of direction and distance to the feature to which the info-mark relates. This would then allow the user to make a more accurate assessment as to where in the environment they are located.

One final item to note is that the sample size used in this study was relatively small with 30 participants in total and thus 10 in each group. The use of the smaller sample size was due to a number of logistical constraints which limited the number of trials that could be conducted. Therefore it should be noted that although the statistical testing methods implemented within this study are suitable for such small sample sizes, larger scale studies may find different results.

\section{Future Work}

This study has presented a method for providing additional information to a user in the form of info-marks as a means of attempting to improve navigational performance in a rural VE. 
Although the results obtained have not shown a particular improvement of implementing such methods, this does not mean that the continuation of investigation is not warranted. For example, it was clear that users felt that the provision of such information improved their performance even though this was not the case. A possible future investigation is to address this paradox and investigate the properties of the info-marks to see if altering them affects the performance of the navigational task (i.e. altering the distance from the feature at which the information is presented). Another investigation would be to see if the same results are obtained in a similar implementation in a real world environment. Obviously in that case it might be impractical to cover such large distances, but a smaller scale implementation could shed light on the transferability of techniques. A further investigative topic for consideration is with regards to the interaction with the environment and how this could affect results. In the environment implemented, the user moved using mouse interactions which provided no proprioceptive feedback. Such feedback (through interfaces such as treadmills) may affect the impact of the information provided. In addition, impact detection was not implemented, meaning that the user could cross rivers, walk through buildings and climb steep slopes, all of which could impact on the navigational performance as a result of mimicking the real world more closely.

\section{References}

Abu-Obeid N 1998 Abstract and scenographic imagery: The effect of environmental form on wayfinding. Journal of Environmental Psychology 18: 159-73

Aoki H, Oman C M, Buckland D A, and Natapoff A 2008 Desktop-VR system for preflight 3D navigation training. Acta Astronautica 63: 841-47

Ardito C, Costabile M F, De Angeli A and Pittarello F 2007 Navigation help in 3D worlds: Some empirical evidences on use of sound. Multimedia Tools and Applications 33: 201-16

Biggs M, Fischer U, and Nitsche M 2008 Supporting wayfinding through patterns within procedurally generated virtual environments. In Proceedings of the ACM SIGGRAPH Symposium on Video Games, Los Angeles, California: 123-28

Bosco A, Picucci L, Caffò A O, Lancioni G E, and Gyselinck V 2008 Assessing human reorientation ability inside virtual reality environments: The effects of retention interval and landmark characteristics. Cognitive Processing 9: 299-309

Brosset D, Claramunt C, and Saux E 2008 Wayfinding in natural and urban environments: A comparative study. Cartographica 43: 21-30

Burigat S and Chittaro L 2007 Navigation in 3D virtual environments: Effects of user experience and locationpointing navigation aids. International Journal of Human Computer Studies 65: 945-58

Byagowi A and Moussavi Z 2012 Design of a virtual reality navigational (VRN) experiment for assessment of egocentric spatial cognition. In Proceedings of the Annual International Conference of the IEEE Engineering in Medicine and Biology Society, San Diego, California: 4812-15

Cauchard J R, Löchtefeld M, Irani P, Schoening J, Krüger A, Fraser M, and Subramanian S 2011 Visual separation in mobile multi-display environments. Proceedings of the Twenty-fourth Annual ACM Symposium on User Interface Software and Technology, Santa Barbara, California: 451-60

Darken R P and Peterson B 2001 Spatial orientation, wayfinding, and representation. In Stanney K (ed) Handbook of Virtual Environment Technology. Hillsdale, NJ, Lawrence Erlbaum: 493-518

Fillatreau P, Fourquet J, Le Bolloc'h R, Calilhol S, Datas A, and Puel B 2013 Using virtual reality and 3D industrial numerical models for immersive interactive checklists. Computers in Industry 64: 1253-62

Fogli D, Pittarello F, Celentano A, and Mussio P 2003 Context-aware interaction in a mobile environment. In Proceedings of the Fifth International Symposium of Mobile HCI, Human-Computer Interaction with Mobile Devices and Services, Udine Italy: 434-39

Giudice N A, Bakdash J Z, Legge G E, and Roy R 2010 Spatial learning and navigation using a virtual verbal display. ACM Transactions on Applied Perception 7: 3-22

Grant S C and Magee L E 1998 Contributions of proprioception to navigation in virtual environments. Human Factors 40: 489-97

Hegarty M, Richardson A E, Montello D R, Lovelace K, and Subbiah I 2002 Development of a self-report measure of environmental spatial ability. Intelligence 30: 125-47 
Hurlebaus R, Basten K, Mallot H A, and Wiener J M 2008 Route learning strategies in a virtual cluttered environment. In Proceedings of the International Conference on Spatial Cognition VI: Learning, Reasoning, and Talking about Space, Freiburg, Germany: 104-20

Kettunen P, Irvankoski K, and Krause C M 2013 Landmarks in nature to support wayfinding: The effects of seasons and experimental methods. Cognitive Processing 14: 245-53

Klippel A and Winter S 2005 Structural salience of landmarks for route directions. In Cohn A G and Mark D M (eds) Spatial Information Theory: International Conference, COSIT 2005, Ellicottville, NY, USA, September 14-18, 2005, Proceedings. Berlin, Springer-Verlag Lecture Notes in Computer Science, Vol. 3693: 347-62

Koenig S, Crucian G, Dalrymple-Alford J, and Dünser A 2011 Assessing navigation in real and virtual environments: A validation study. International Journal on Disability and Human Development 10: 325-30

Kühn P J 2004 Location-based services in mobile communication infrastructures. International Journal of Electronics and Communications 58: 159-64

Lathrop W B and Kaiser M K 2005 Acquiring spatial knowledge while traveling simple and complex paths with immersive and non-immersive interfaces. Presence: Teleoperators and Virtual Environments 14: 249-63

Lazem S Y and Sheta W M 2005 Automatic landmark identification in large virtual environment: A spatial data mining approach. In Proceedings of the Ninth International Conference on Information Visualisation, London, United Kingdom: 752-57

Lynch K 1960 The Image of the City. Cambridge, MA, MIT Press

Lyu S, Lin Y, Huang S, and Yau J 2013 Experience-based virtual training system for knee arthroscopic inspection. BioMedical Engineering Online 12(1): 63

Montello D R 2005 Navigation. In Shah P and Miyake A (eds) The Cambridge Handbook of Visuospatial Thinking. New York: Cambridge University Press: 257-94

Parush A and Berman D 2004 Navigation and orientation in 3D user interfaces: The impact of navigation aids and landmarks. International Journal of Human Computer Studies 61: 375-95

Pierce J S and Pausch R 2004 Navigation with place representations and visible landmarks. In Proceedings of the IEEE Virtual Reality Conference, Chicago, Illinois: 173-80

Rashid U, Nacenta M A, and Quigley A 2012a The cost of display switching: A comparison of mobile, large display and hybrid UI configurations. In Proceedings of the International Working Conference on Advanced Visual Interfaces, Capri Island, Italy: 99-106

Rashid U, Nacenta M A, and Quigley A 2012b Factors influencing visual attention switch in multi-display user interfaces: A survey. In Proceedings of the 2012 International Symposium on Pervasive Displays, Porto, Portugal

Raubal M and Winter S 2002 Enriching wayfinding instructions with local landmarks. In Proceedings of the Second International Conference of GIScience, Boulder, Colorado: 243-59

Rousell A J, Jarvis C H, and Li J 2008 The effectiveness of artificial landmarks for orientation tasks within a large-scale virtual environment. In Proceedings of the Sixteenth Annual GIS Research UK Annual Conference, Manchester, United Kingdom

Ruddle R A and Péruch P 2004 Effects of proprioceptive feedback and environmental characteristics on spatial learning in virtual environments. International Journal of Human Computer Studies 60: 299-326

Ruddle R A, Volkova E, and Bülthoff H H 2013 Learning to walk in virtual reality. ACM Transactions on Applied Perception 10(2): 11

Siegel A W and White S H 1975 The development of spatial representations of large-scale environments. Advances in Child Development and Behaviour 10: 9-55

Sjölinder M, Höök K, Nilsson L, and Andersson G 2005 Age differences and the acquisition of spatial knowledge in a three-dimensional environment: Evaluating the use of an overview map as a navigation aid. International Journal of Human Computer Studies 63: 537-64

Snowdon C and Kray C 2009 Exploring the use of landmarks for mobile navigation support in natural environments. In Proceedings of the Eleventh International Conference on Human-Computer Interaction with Mobile Devices and Services, Bonn, Germany

Sorrows M E and Hirtle S C 1999 The nature of landmarks for real and electronic spaces. In Proceedings of the International Conference on Spatial Information Theory: Cognitive and Computational Foundations of Geographic Information Science, Stade, Germany: 37-50

Sturz B R, Bodily K D, Katz J S, and Kelly D M 2009 Evidence against integration of spatial maps in humans: Generality across real and virtual environments. Animal Cognition 12: 237-47

Thibault G, Pasqualotto A, Vidal M, Droulez J, and Berthoz A 2013 How does horizontal and vertical navigation influence spatial memory of multi-floored environments? Attention, Perception, and Psychophysics 75: $10-5$ 
Vinson N G 1999 Design guidelines for landmarks to support navigation in virtual environments. In Proceedings of the SIGCHI Conference on Human Factors in Computing Systems, Pittsburgh, Pennsylvania: $278-85$

Vinson N G, Lapointe J, Parush A, and Roberts S 2012 Cybersickness induced by desktop virtual reality. In Proceedings of the Graphics Interface Conference, Toronto, Ontario: 69-75

Yaegashi K and Yanai K 2009 Can geotags help image recognition? In Wada T, Huang F, and Lin S (eds) Advances in Image and Video Technology: Third Pacific Rim Symposium, PSIVT 2009, Tokyo, Japan, January 13-16, 2009, Proceedings. Berlin, Springer Lecture Notes in Computer Science Vol. 5414: 361-73 